\title{
Improvement of patient satisfaction with the neurosurgery service at a large tertiary care, London-based hospital
}

\author{
Amad Khan, Mohammad Naushad Chaudhry, Salema Khalid, Dipankar Nandi \\ Charing Cross Hospital, Imperial College Healthcare NHS Trust
}

\begin{abstract}
Patient satisfaction is central to healthcare provision and the effective running of any surgical unit. Following on from both formal and informal feedback, we decided to look objectively at patient satisfaction with the neurosurgery service at a large tertiary care hospital in London and identify areas that needed improvement within the unit. Patient satisfaction was looked at with respect to four different aspects of the neurosurgery service: the surgeons, ward doctors, nurses, and hospital services. A questionnaire-based cross-sectional study was conducted and once the data were collected a plan of action to improve service provision was put into place. Data were collected from 150 patients over a 3 month period from September to November 2012. Interventions were made and data re-collected from 150 patients from January to March 2013. With regards to satisfaction with the neurosurgery service, $76.7 \%(n=115)$ were satisfied; following implementation of our measures for improvement, which included staff education, meetings and posters, this figure increased to $90.6 \%$ ( $n=136, p<0.001$ on Chi-square testing). In conclusion, patient satisfaction should be at the crux of patient care, with a strong focus on effective communication skills, and can be improved by identification of issues by direct patient feedback and subsequent action based on this.
\end{abstract}

\section{Problem}

Both formal and informal feedback from patients under the care of the neurosurgery service at our hospital had shown some areas of concern that needed improvement. While the 'Friends and Family Test' was in place at our unit, it only asked how likely patients would recommend our service to their loved ones and did not go into specific areas of concern or excellence (1). Furthermore, there is a formal complaints process but this is only used by patients when a serious mishap occurs. Always striving for excellence, we decided to take matters into our own hands and obtain feedback directly from patients and try to improve our service. We aimed to ask patients specific questions about four areas: the surgeons, ward doctors, nurses, and the hospital services. We asked especially pertinent and often neglected questions in feedback forms which focused on the communications skills of the staff, their bedside manner, and the confidence the patients had of being in their care. These questions would help us to identify and quantify any shortcomings objectively, making quality improvement possible. Our study was based at the neurosurgery inpatient wards at a large tertiary care hospital in London, UK.

\section{Background}

The NHS in the UK has customarily been regarded as a system under great financial and resource pressures. Consequently, when patients are questioned formally, they have tended to excuse dissatisfying aspects of their care, for reasons of gratitude to the system and the persons involved (2). However, the 'reliability' (replicability score) of patient satisfaction surveys has been shown to be high and the value of surveys using elementary satisfaction 'scales' (e.g. 'very satisfied' through to 'very dissatisfied') lies in their (repeated) use across different hospital settings on specific patient experiences and procedures (3).

No healthcare system is free from flaws and there is always need for improvement. Certain cross-sectional studies have looked at patient satisfaction with the NHS, but none specifically regarding neurosurgery, a tertiary specialist field with relatively small patient numbers. Our objective was to look at patient satisfaction with the neurosurgery service at a large tertiary care hospital in London and identify areas that needed to be addressed and improved within this NHS service. This concept of review and change is designed to achieve improved clinical standards and quality of patient care (4).

\section{Baseline measurement}

We used the Plan-Do-Study-Act (PDSA) model of quality improvement. Our first PDSA cycle acted like a pilot study and helped us gather initial data which were useful in gauging the scale of the problem.

In PDSA cycle 1 we handed out 30 questionnaires to patients on the neurosurgery ward and asked them to give us feedback on all aspects of their care that they felt were lacking. There were no leading questions used, but instead blank comment boxes so as to let the patients express who and what was important to them for their care on the ward. It was on the basis of the data from our first PDSA that we refined our questionnaire and came up with the aforementioned four categories (surgeons, ward doctors, nurses, hospital services) and used the comments as the basis for our questions (figure 1: Questionnaire).

In PDSA cycle 2 we gathered the data; we gave out and collected 150 anonymised questionnaires over a period covering 3 months from September to November 2012. With regards to satisfaction with the neurosurgery service overall, $76.7 \%(n=115)$ of 
respondents said they were satisfied.

See supplementary file: ds3081.pdf - "Questionnaire"

\section{Design}

All the patients admitted to the neurosurgery wards at this hospital from September to November 2012 were included in our sample and thus were given the questionnaires. Our sample size was 150 and we achieved a response rate of $100 \%$. The questionnaire was given to the patients in the neurosurgery wards and completed by them at the time of their discharge from the neurosurgery service. If they were unable to complete it, their carer was requested to do so.

The questionnaire was based on questions to assess the level of satisfaction of inpatients with the neurosurgery service. It was divided into questions pertaining to the neurosurgeons (both consultants and registrars), ward doctors (house and senior house officers), nursing staff, hospital services, and finally some demographic questions.

All questions were phrased to ask whether the patients were satisfied with the care provided and had four possible responses: 'not at all', 'no', 'yes to a certain extent', and 'yes completely'. A score of 4 was given for 'yes completely', 3 for 'yes', and 2 for 'no', while 'not at all' was given a score of 1 . A respondent was considered to be satisfied overall when he or she had a score of $75 \%$ or more of the total possible points on the questionnaire. All data entry and analysis was done using SPSS version 17.0

\section{Strategy}

Once the data had been collected from our second PDSA cycle, we analysed it and produced some recommendations. The recommendations were based on individual comments given by patients and more importantly on questions that identified a deficiency by virtue of more than $20 \%$ of respondents answering either 'no' and 'not at all'.

For the neurosurgeons, the feedback was that there should be more patient involvement in discussions of treatment and increased attention and time given to listening to what patients have to say. For the ward doctors there was again room for improvement in communication skills; there was a need for them to give more understandable explanations to questions posed by the patients. They were also urged to improve communication with doctors from other services involved in the patients' care. The feedback for the nurses was that they should prevent misinformation from being given to patients with regards to their illness and their future course of stay in the ward. Finally, there was a consensus for a need for more high dependency unit (HDU) beds and better quality food regarding the services provided by the hospital (table 1).

Armed with this information our third PDSA cycle set about involving the right stakeholders and getting their point of view on the matter. First, we sent an email to all the staff members detailing the work we had done and the deficiencies identified. Our next step was to put up posters with the suggestions for each group mentioned above in all the relevant wards. We then arranged two separate meetings - the first after liaising with the matron and aimed at the nursing staff. The results of PDSA cycle 2 were discussed and an open and honest discourse with the nursing staff ensued. The deficiencies identified were put forward to the nursing staff and a brainstorming session ensued in which they were asked to come up with solutions. The main complaint from patients was the provision of wrong information. The nurses came up with an effective way around this problem: the most senior nurse on duty would join the ward round with the doctors every day and handover to the nursing staff so as to prevent miscommunication. The second meeting was with the surgeons (consultants and registrars) and the ward doctors (house and senior house officers) where a similar process followed. This forum was interesting as it brought out experiences that the doctors had had with patients - both good and bad. In order to help with deficiencies with communication skills, we liaised with the medical education department and arranged for a communication skills session for the junior doctors. This was not just a one-off but was incorporated into the foundation doctors' regular compulsory teaching schedule, thus ensuring continuity for years to come. Finally, in order for there to be improvement in the logistics department, the relevant findings were forwarded by the matron to the concerned departments.

The post-intervention data collection was carried out after 2 months. The data was gathered over a period of 3 months i.e. from January to March 2013.

\section{Results}

Overall there were similar numbers of males (53\% and $51 \%)$ and females ( $47 \%$ and $49 \%$ ) for both the pre- and post-intervention data, respectively. Furthermore, the patients were representative of the demographics of the area served by the hospital with a median age group of 51-65 years (5).

The percentage of patients who answered 'yes, completely' to the questions pertaining to satisfaction with the surgeons increased from $58.7 \%$ to $67.3 \%$, which though impressive was not statistically significant on chi-square testing $(\mathrm{p}=0.12)$. The percentage of the patients who were completely satisfied with the care provided by the junior doctors on the ward improved from $67.3 \%$ to $82 \%$, which was statistically significant $(p<0.05)$. Similarly, complete satisfaction with the care provided by the nurses increased from $71.3 \%$ to $89.3 \%$, which was highly significant $(p<0.001)$. Satisfaction with the services of the hospital only improved from $58 \%$ to $66 \%$ and was not statistically significant $(p=0.15)$. Figure 2 (graph 1$)$ shows the improvement in the number of patients pre- and post-intervention broken down into the four care provision groups.

Overall satisfaction with the neurosurgery service was calculated by using the number of patients who had a score of $75 \%$ or more as detailed in the Design section. Overall, with regard to satisfaction with the neurosurgery service, $76.7 \%(n=115)$ were satisfied; following implementation of our measures for improvement, this figure increased to $90.6 \%(n=136)(p<0.001$ on Chi-square testing). An often overlooked aspect of questionnaires is the comments 
section. Table 1 contains excerpts and sums up the comments section from all 300 questionnaires in terms of strengths and weaknesses. As mentioned before, these comments were invaluable in helping us design the improvement strategy we implemented.

See supplementary file: ds3249.pdf - "Graph of patients vs care provision groups and table of comments"

\section{Lessons and limitations}

Regular critical analysis of current methods of practice in medicine is essential to maintain high quality clinical care to our patients. Previously set standards need to be periodically reviewed and occasionally altered to achieve this objective. Medical audit - by setting guidelines, appraising results, and implementing changes is an invaluable tool for this purpose (6). The Australian Clinical Review (1981-7), in analysing 71 clinical audit studies, had the potential to identify problems and therefore to induce change and improvement (7). Audit, therefore, is an instrument for change, the organisation of which can be made as simple as the tasks involved would allow. Quality improvement projects go a step further and have the potential to alter and strengthen other aspects of hospital practice and organisation, our project being a prime example of such a situation.

A higher proportion of patients who used hospitals in England in 2007 were satisfied with the care they received than in the previous year, a Healthcare Commission survey showed (8). The study, carried out by the Picker Institute, looked at responses from nearly 76000 inpatients treated at a total of 165 English hospital trusts and, to date, is the largest of its kind. It showed that nearly all patients $(92 \%)$ rated their care as good, very good, or excellent. In our study, $90.6 \%$ of the total number of patients gave positive responses about the neurosurgery care provided post-intervention, which is slightly less, but comparable nonetheless.

The lessons learnt from this project are that simple cost-effective measures such as working on communication skills and continual reminders for improvement based on patient feedback can work wonders when it comes to improving patient satisfaction.

The challenges faced by our team were mainly logistical in nature. Getting all the stakeholders in one place was at times challenging. Also, certain factors identified from the patient feedback could not be dealt with immediately, especially points like the need for more interpreters and more HDU beds. The limitation for these weaknesses was obviously cost, but we nonetheless forwarded them to the relevant departments for consideration. If we were to repeat the study, we would try to get more staff involved in data collection which would facilitate the process manifold. A limitation which may affect our results is the fact that getting patients to fill in questionnaires would bias the results, as patients would feel obliged to be more appreciative so as to not compromise their care. We tried to overcome this by making sure we let patients know that their answers would be confidential and would not affect their care. Furthermore, we let patients fill in the forms in their own time and not while we were around, in order not to pressurise them in any way. Another limitation was that some of the junior doctors changed over the course of the study. The flip side to this, however, is that two sets of junior doctors were directly involved in improvement of patient satisfaction and helped promulgate the message of this quality improvement plan among a larger number of colleagues.

Our sample size was sufficient and a true representation of our monthly average intake. Our study was also cost-effective in that the only expenditure was related to printing out the questionnaires. In order to maintain continuity, the communication skills sessions have become a regular part of the junior doctors' mandatory teaching. Furthermore, we have entrusted our junior colleagues to repeat a similar study in the department on a yearly basis so as to continually identify areas for improvement and not get complacent.

\section{Conclusion}

After comparing the data in our study with those of other studies, and bearing in mind our hospital is a tertiary care centre run by the NHS, we feel that our findings are generalisable to other NHS tertiary care centres in the UK. In conclusion, our study shows that an effective way to bring about improvement in patient satisfaction is through simple measures such as direct patient feedback and continual reminders for better communication skills.

\section{References}

1. http://www.imperial.nhs.uk/patients/patient_experience/frien dsandfamilytest/index.htm

2. Philip M, Gillham NR. NHS provision for the treatment of ankle fractures: a patient satisfaction study. J R Soc Med 1993;86:332-5.

3. Wickings I. Proof of the Pudding. Hlth Services J 1989; 31 August:1070-1 [see the discussion of the methodology adopted by the CASPE Unit (Clinical Accountability, Service Planning and Evaluation)]

4. Delacey G. What is audit? Why should we be doing it? Hosp Update 1992;18:458-66.

5. http://www.lbhf.gov.uk/Directory/Council and Democracy/PI ans_performance_and_statistics/Statistics_and_census inf ormation/Census information/174025_2011_Census_Popul ation Estimates.asp

6. Murthy P, Mckerrow WS. Routine nasal surgery: an audit of outpatient follow-up. Postgrad Med J 1996;72:37-40C.

7. Collopy BT. Audit activities in Australia. BMJ 1991;303:1523.

8. Eaton L. NHS sees a steady rise in patient satisfaction over five years. BMJ 2008;336:1091.

\section{Declaration of interests}

None.

\section{Acknowledgements}

We would like to thank the support staff on the wards for their help 
and co-operation. 